\title{
What it costs to implement a plan: Plan-level and task-level contributions to switch costs
}

\author{
GORDON D. LOGAN \\ Vanderbilt University, Nashville, Tennessee
}

\begin{abstract}
Four experiments were conducted to identify the costs of implementing a plan in the task span procedure, which requires subjects to retrieve the task to perform on the current target from a list of planned tasks in memory. Experiment 1 compared switch costs in the task span procedure with switch costs in the explicit taskcuing procedure, which presented cues indicating the task to perform on each target. Switch costs were greater in the task span procedure. Experiments 2-4 were designed to identify the sources of this difference. Experiment 2 showed that the requirement of establishing a correspondence between the list of task names and the list of targets contributed to switch costs. Experiment 3 showed that retaining lists of similar task names produced greater switch costs than did retaining lists of dissimilar task names. Experiment 4 showed that memory load had no effect on switch costs. The results are discussed in terms of the interaction between plan-level and task-level processing in the implementation of plans.
\end{abstract}

Plans play an important role in executive control of thought and action, allowing people to behave in a coherent fashion by coordinating separate activities and directing them toward a common goal (Miller, Galanter, \& Pribram, 1960). These benefits of planning come with a cost. The plans must be created or interpreted from instructions, and that takes time and effort. Most of the research on planning has been concerned with the processes that produce these costs (e.g., Hayes-Roth \& Hayes-Roth, 1988; Kotovsky, Hayes, \& Simon, 1985; Thomas, 1974). The present article is concerned with the costs that result from implementing a plan. As plans are implemented, some executive processes must be directed toward the plan itself and away from the subordinate processes that accomplish the steps in the plan. The former, plan-level processes may interact with the latter, task-level processes, increasing the time to accomplish each step and the time to switch from one step to another. The purpose of the present article is to investigate the interaction between plan-level and tasklevel processes in order to identify the costs involved in implementing a plan.

Plans consist of a series of subordinate goals, or subgoals, to be obtained in the service of an overarching goal (Miller et al., 1960). In some cases, the subgoals are directly related to each other, dividing the activity into parts that must be combined to accomplish the overarching goal, such as the steps involved in preparing a meal. In other cases, the subgoals are unrelated to each other and contribute independently to the accomplishment of the overarching goal, such as a list of jobs to be done on a workday. The present experiments focus on the task span procedure, which provides a laboratory analogue of the implementation of the second kind of plan. The procedure, illustrated in Figure 1, involves a study phase, in which subjects are presented with a list of tasks to be accomplished, followed by a test phase, in which subjects are presented with a series of target stimuli on which tasks must be performed. In Figure 1, the tasks involve making parity (odd-even) or magnitude (higher or lower than 5) judgments on a series of digits. The list of task names is analogous to a plan, in that it specifies what subjects are supposed to do with the subsequent series of targets. In order to implement the plan, subjects must maintain the list in memory, step through the list as they progress through the series of targets, and coordinate each task on the list with the corresponding target in the series. In the course of implementing the plan, subjects must switch from one task to another, and these switches produce robust increases in reaction time (RT) and reductions in accuracy, known as switch costs (Logan, 2004, 2006).

Switch costs in the task span procedure are larger than switch costs in other task-switching procedures (for reviews, see Logan, 2003; Monsell, 2003), and the purpose of the present experiments was to determine why. The main hypothesis was that the task span procedure involves plan-level processes that interact with the task-level processes in ways that increase the magnitude of switch costs. The experiments examined the effects of three kinds of plan-level processes on task-level switch costs: (1) keeping track of the order of tasks and retrieving the next one

G. D. Logan, gordon.logan@vanderbilt.edu 


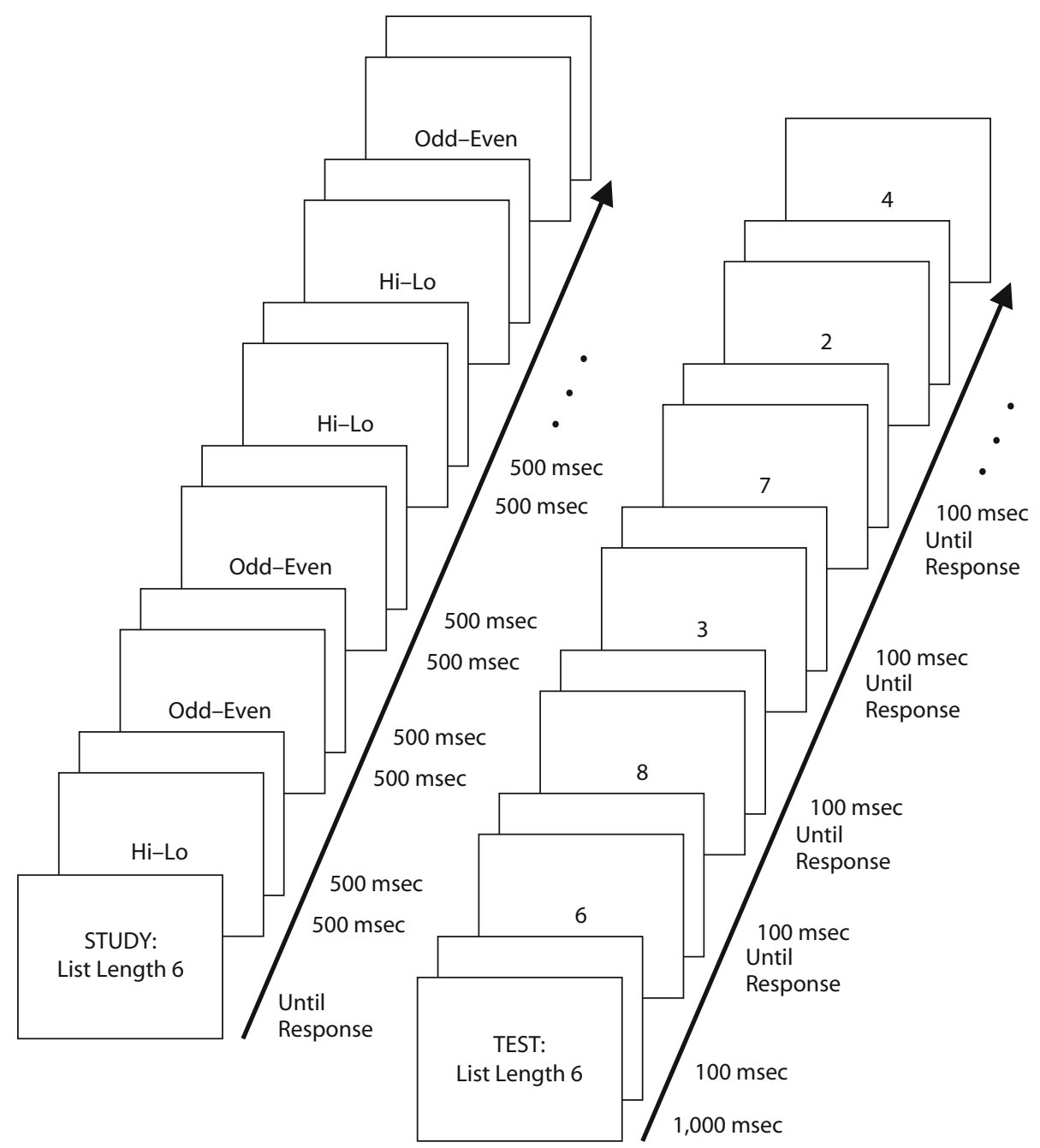

Figure 1. The events on the study and test phases of the task span procedure in the present experiments. The events in the recall phase are not shown. The study phase begins with a warning display that is exposed until the subject presses a key. Then the list of task names is presented at a rate of one per second; each task name is exposed for $500 \mathrm{msec}$, followed by a 500 -msec blank screen. Then the warning display for the test phase is displayed for $1,000 \mathrm{msec}$. It is followed by a $100-\mathrm{msec}$ blank screen, after which the first target is presented. Targets are presented until the subject responds, and each response is followed by a 100-msec blank screen. The events in the study and the test phases of the explicit task-cuing conditions are the same, except that a cue indicating which task to perform is presented with each target in the test phase.

in the plan, (2) protecting task-level processing from content-specific interference from elements of the plan and vice versa, and (3) maintaining the list of tasks in working memory.

In the first experiment, switch costs in the task span procedure were compared directly with switch costs in the explicit task-cuing procedure (Meiran, 1996; Sudevan \& Taylor, 1987), which does not require implementation of a plan. The subsequent experiments were designed to build a procedural bridge between the task span procedure and the explicit task-cuing procedure by requiring subjects to retain a list of tasks in memory while performing explicitly cued task switching. The nature of the lists was varied within and between experiments to create possible sources of the interaction between plan-level and task-level pro- cesses and to evaluate their effects on the magnitude of task-level switch costs. The discussion of the experiments will focus primarily on the effects of keeping track of order, of dealing with content-specific interference, and of memory load on RTs and switch costs. Broader theoretical interpretations of the effects will be deferred to the General Discussion section.

\section{EXPERIMENT 1 Task Span Versus Explicit Cuing}

Experiment 1 was designed to compare switch costs in the task span and explicit task-cuing procedures within subjects in order to establish the basic effects to be examined in the subsequent experiments. There were three 
conditions: a task span condition, a cued condition, and a loaded condition. The task span condition was the same as that described above (see Figure 1), except that it required a third, final recall phase after the test phase, in which subjects were asked to recall the task names in order, so that their recall of the plan could be compared with recall in the cued and loaded conditions. The cued condition was the same as the task span condition, except that a cue indicating which task to perform appeared with each target in the test phase. Subjects received a list of task names in the study phase and a list of targets and cues in the test phase. After completing the test phase, subjects recalled the list of task names in a final recall phase. The cues in the test phase were the same task names that were presented in the study phase, and they appeared in the same order. The loaded condition was like a typical explicit task-cuing procedure with an irrelevant memory load, which the subjects recalled in the final recall phase after the test phase. The procedure was like that in the cued condition, except that the memory load was a list of unrelated task names presented in an order that was not predictive of the order in which the cues appeared in the test phase (e.g., subjects received a list of task names consisting of wide-narrow and red-green and then completed a test list of digits using hi-lo and odd-even as cues).

The three conditions differed in the amount of planlevel processing they required and, consequently, in the opportunities they provided for interactions between plan-level and task-level processing. Each condition required subjects to retain a plan in memory-a list of task names to be recalled in the final recall phase-but they differed in the relation between the plan and the tasks to be performed in the retention interval. In the task span condition, the plan was directly relevant. It specified the tasks to be performed on the target stimuli. Subjects had to retrieve task names from the list in order and combine them with the corresponding targets to produce appropriate responses. Moreover, the tasks on the list were the same tasks as those that were performed on the targets, so subjects had to protect the current task from contentspecific interference from other tasks on the list and protect the list against content-specific interference from the current task. Ordered retrieval and protection from interference might inflate switch costs, relative to the other conditions. In the cued condition, subjects did not need to retrieve task names, but they might need to protect themselves from content-specific interference between the task names on the list and the cues presented with the targets. Protection from interference might inflate switch costs, relative to the loaded condition. In the loaded condition, subjects did not need to retrieve task names, nor did they need to protect themselves from contentspecific interference. Switch costs should be smallest here, inflated only by the requirement to retain the plan for final recall in memory, which was common to the other conditions.

In order to equate memory loads in the three conditions, the experiment used two sets of tasks. One set consisted of magnitude and parity judgments of digits, using $h i-l o$ and odd-even as cues. The other set consisted of width and color judgments of rectangles, using wide-narrow and red-green as cues. Wide-narrow and red-green were used to generate memory loads in the loaded condition when subjects performed the magnitude and parity tasks. $\mathrm{Hi}-\mathrm{lo}$ and odd-even were used to generate memory loads in the loaded condition when subjects performed the width and color tasks. Thus, across conditions, the same items were used in the memory loads.

\section{Method}

Subjects. Twelve subjects from the general university population served in two 1-h sessions for pay.

Apparatus and Stimuli. The stimuli were displayed on Sony Trinitron monitors controlled by Dell Dimension computers. The stimuli for the magnitude and parity tasks were the task names hi-lo and odd-even and the digits $1,2,3,4,6,7,8$, and 9, presented in the center of the screen. In the test phase of the task span condition, each digit appeared alone in the center of the screen. In the test phase of the cued and loaded conditions, the appropriate task name appeared as a cue one row above the digit. Each letter and digit was $7 \mathrm{~mm}$ high $\times 4 \mathrm{~mm}$ wide. The task names for the width and color tasks were wide-narrow and red-green, and the target stimuli were red and green rectangles that were $1.2 \mathrm{~cm}$ high and $1.4 \mathrm{~cm}$ (wide stimuli) and $0.5 \mathrm{~cm}$ (narrow stimuli) wide. In the test phase of the task span procedure, each rectangle appeared alone in the center of the screen. In the test phase of the cued and loaded conditions, the appropriate task name appeared as a cue immediately above the rectangle.

The study phase of each set of trials was preceded by a warning display that said "STUDY PHASE: 6 trials," and the test phase was preceded by a warning display that said "TEST PHASE: 6 trials." The recall phase began with a warning prompt that said "RECALL: 6 trials" and was followed by recall prompts that consisted of three centered asterisks (i.e., ${ }^{* * *}$ ). Viewing distance was not controlled but was approximately $60 \mathrm{~cm}$. At this distance, $1 \mathrm{~cm}$ on the screen corresponds to approximately $1^{\circ}$ of visual angle. There were 20 different lists of task names, presented in Table 1, which included all possible orderings of three sets of two task names. Responses were collected from the numeric keypad on the computer keyboard.

Procedure. The events in the study and test phases of the task span procedure are depicted in Figure 1. Each set of trials began with a warning display indicating the study phase. It was displayed until subjects pressed a key on the computer keyboard. Then the six task names were presented at a rate of one per second. Each name was exposed for $500 \mathrm{msec}$, followed by a blank screen for $500 \mathrm{msec}$. Immediately after the blank period following the last task name, a warning display appeared, indicating the test phase. It was exposed for $1,000 \mathrm{msec}$ and was followed by a 100 -msec blank screen. Then a target - a single digit or a colored rectangle - was exposed until the subject responded. Then the screen went blank for a 100 -msec response-to-stimulus interval, and the next target was presented.

Table 1

Lists of Task Names Used in the Experiments

\begin{tabular}{ccccc}
\hline $\begin{array}{c}\text { Original } \\
\text { Sequence }\end{array}$ & $\begin{array}{c}\text { Opposite } \\
\text { Sequence }\end{array}$ & $\begin{array}{c}\text { Sequential } \\
\text { Effect }\end{array}$ & $\begin{array}{c}\text { Number of } \\
\text { Repetitions }\end{array}$ & $\begin{array}{c}\text { Number of } \\
\text { Alternations }\end{array}$ \\
\hline HHHOOO & OOOHHH & RRARR & 4 & 1 \\
HHOOOH & OOHHHO & RARRA & 3 & 2 \\
HOOOHH & OHHHOO & ARRAR & 3 & 2 \\
HHOHOO & OOHOHH & RAAAR & 2 & 3 \\
HHOOHO & OOHHOH & RARAA & 2 & 3 \\
HOHHOO & OHOOHH & AARAR & 2 & 3 \\
HOOHHO & OHHOOH & ARARA & 2 & 3 \\
HOHOOH & OHOHHO & AAARA & 1 & 4 \\
HOOHOH & OHHOHO & ARAAA & 1 & 4 \\
HOHOHO & OHOHOH & AAAAA & 0 & 5 \\
\hline
\end{tabular}

Note- $\mathrm{H}$, hi-lo task; O, odd-even task; R, repetition; A, alternation. 
This continued until all six targets had been presented. Then the warning display for the recall phase was presented for $1,000 \mathrm{msec}$ and was followed by a blank display for $100 \mathrm{msec}$. Next the recall prompt appeared until the subject responded. Then the screen was blank for a 100-msec response-to-stimulus interval until the next recall prompt appeared.

In the task span condition, subjects were told to remember the six task names on each study list and to perform each task on the list on the corresponding target in the test phase as quickly and accurately as possible by pressing the 1 and 3 keys on the computer keyboard. Then they were told to recall the task names in order as accurately as possible by pressing the 7 and 9 keys in response to the prompts in the recall phase. When performing the magnitude and parity tasks in the test phase, all subjects pressed the 1 key on the numeric keypad for odd and high and the 3 key for even and low. In the recall phase, all subjects pressed the $7 \mathrm{key}$ for hi-lo and the 9 key for odd-even. When performing the color and width tasks, all subjects pressed the 1 key to report wide and red and the 3 key to report narrow and green. In the recall phase, all subjects pressed the 7 key to report the width task and the 9 key to report the color task.

The procedure for the cued condition was the same as the procedure for the task span condition, except that cues indicating which task to perform ( $h i-l o$ or odd-even for the magnitude and parity tasks; wide-narrow or red-green for the width and color tasks) appeared centered in the row above the target stimulus. The order in which the cues appeared in the test phase was the same as the order in which the task names had appeared in the study phase.

The procedure for the loaded condition was the same as the procedure for the cued condition, except that the task names presented in the study phase described a different set of tasks from those described by the cues presented in the test phase and appeared in an order that was different from the order of tasks in the test phase. Subjects who performed the parity and magnitude tasks in the test phase had the task names for the width and color tasks in the study phase; subjects who performed the width and color tasks in the test phase had the task names for the parity and magnitude tasks in the study phase.

There were 40 study-test blocks in each condition in each session, in which each of the 20 lists in Table 1 was presented twice, in random order. The order of targets in the test phase was randomized separately for each test block for each subject. Subjects performed all three conditions with one set of test phase tasks in one session and then performed all three conditions with the other set of test phase tasks in the other session. Half of the subjects had the parity and magnitude tasks in the first session and the width and color tasks in the second session. Half of the subjects had the opposite. There were six possible orders of the three conditions (i.e., TCL, TLC, CLT, CTL, LTC, and LCT, where T, C, and L represent the task span, cued, and loaded conditions, respectively). Two subjects received the conditions in each of these orders, and each subject received the conditions in the same order in each session. One of the 2 subjects receiving each order of conditions had the parity and magnitude tasks before the width and color tasks, and the other had the opposite.

\section{Results}

Test phase. Mean RTs were calculated for correct responses to repetitions and alternations in Serial Positions 2-6 in each condition for each set of tasks for each subject. The first serial position was excluded because it could not be classified as a repetition or an alternation. The means across subjects and tasks are presented in Figure 2. The percentage of correct responses in each condition is inset on the corresponding bar in the figure. Accuracy was high, and there was no evidence of a speed-accuracy trade-off.

Overall, subjects performed the width and color tasks on the rectangles $106 \mathrm{msec}$ faster than they performed the parity and magnitude tasks on the digits, and the difference between repetitions and alternations was smaller with the width and color tasks $(211 \mathrm{msec})$ than with the parity and magnitude tasks $(286 \mathrm{msec})$. Nevertheless, the pattern of performance was the same for the two sets of tasks. The difference between repetitions and alternations was largest in the task span condition (349 msec), intermediate in the cued condition (243 msec), and smallest in the loaded condition (155 msec).

These conclusions were supported by a 3 (condition: task span, cued, or loaded) $\times 2$ (repetition: repetition or alternation) $\times 2$ (tasks: width and color or parity and magnitude) ANOVA on the mean RTs. There were significant main effects of condition $\left[F(2,22)=14.80, M S_{\mathrm{e}}=\right.$ $111,193.32, p<.01]$ and repetition $[F(1,11)=48.49$, $\left.M S_{\mathrm{e}}=45,789.39, p<.01\right]$ and a significant interaction between them $\left[F(2,22)=30.95, M S_{\mathrm{e}}=3,658.53, p<.01\right]$, confirming the differences in switch costs between conditions. Nonorthogonal contrasts showed that switch costs were greater in the task span condition than in the cued condition $\left[F(1,22)=18.43, M S_{\mathrm{e}}=3,658.53, p<.01\right]$, greater in the task span condition than in the loaded condition $\left[F(1,22)=61.72, M S_{\mathrm{e}}=3,658.53, p<.01\right]$, and greater in the cued condition than in the loaded condition $\left[F(1,22)=12.70, M S_{\mathrm{e}}=3,658.53, p<.01\right]$. In addition, there was a significant main effect of task $[F(1,11)=$ 7.08, $\left.M S_{\mathrm{e}}=57,686.77, p<.05\right]$, and a significant interaction between task and repetition $[F(1,11)=8.14$, $\left.M S_{\mathrm{e}}=6,342.58, p<.05\right]$ but the three-way interaction between task, repetition, and condition was not significant $\left[F(2,22)=0.10, M S_{\mathrm{e}}=6,820.94, p=.91\right]$, indicating that the pattern of differences in repetition effects between conditions was the same for the two sets of tasks.

The percentages of correct responses were analyzed in a 3 (condition: task span, cued, or loaded) $\times 2$ (repetition: repetition or alternation) $\times 2$ (tasks: width and color or parity and magnitude) ANOVA. It yielded significant main effects of condition $\left[F(2,22)=5.28, M S_{\mathrm{e}}=16.98\right.$, $p<.05]$ and repetition $\left[F(1,11)=18.99, M S_{\mathrm{e}}=4.03\right.$,

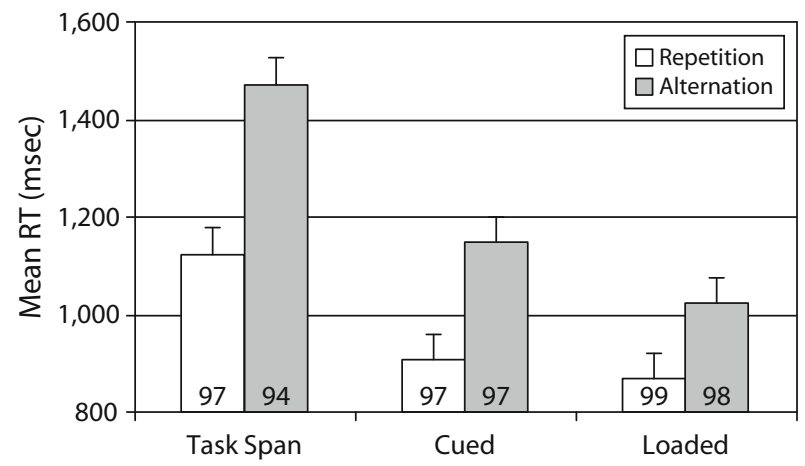

Figure 2. Mean reaction times (RTs) for repetitions and alternations in the task span, cued, and loaded conditions in Experiment 1 . The error bars represent the $95 \%$ confidence intervals for the means, based on Fisher's least significant difference calculated from the interaction between condition, repetition, and task. The numbers inside the bars are the percentage of correct responses in the corresponding condition. 
$p<.01]$ and an interaction between condition and repetition that approached significance $[F(2,22)=3.33$, $\left.M S_{\mathrm{e}}=4.18, p<.06\right]$, consistent with the RT effects. No other effects or interactions were significant.

Recall phase. The percentage of study list items recalled correctly in the final recall phase was calculated for each subject, averaging over serial position. The mean recall percentages were $95.8 \%, 95.5 \%$, and $96.1 \%$ for the task span, cued, and loaded conditions, respectively. A 3 (condition: task span, cued, or loaded) $\times 2$ (tasks: width and color or parity and magnitude) ANOVA on the percentage of correct responses yielded no significant effects.

\section{Discussion}

The differences in switch costs between conditions can be explained in terms of the amount of plan-level processing each condition required. The task span condition required the most plan-level processing. Subjects had to retrieve task names from the list in order and combine them with the corresponding targets, while protecting against content-specific interference from the other task names in memory. The cued condition required protection against content-specific interference but did not require ordered recall during the test phase in order to respond to the targets. However, the correspondence between the memory lists and the sequence of cues may have invited subjects to relate the memory items to the cues anyway. The loaded condition did not require ordered recall or protection against content-specific interference and produced the smallest switch costs. The remaining experiments provided converging evidence for these conclusions.

\section{EXPERIMENT 2 Foreknowledge and Explicit Cuing}

Experiment 2 was designed to evaluate the effects of the correspondence between the order of task names in the study list and of cues in the test list on switch costs; these effects may have increased switch costs in the cued condition in Experiment 1, relative to the loaded condition. The memory lists in the cued and loaded conditions also differed in content; the differences in content were investigated in Experiment 3. To evaluate the effects of order correlation separately from content-specific interference, Experiment 2 compared two cued conditions in which the task names on the study list were the same as the tasks to be performed in the test phase but in which the correspondence between the study phase and the test phase varied. In the relevant condition, the order of task names in the study phase was the same as the order of tasks (and cues) in the test phase. This condition replicated the cued condition in Experiment 1. In the irrelevant condition, the order of task names in the study phase was different from the order of tasks (and cues) in the test phase. The study phase sampled one of the lists in Table 1 at random, and the test phase sampled another. This condition was a mixture of the cued and the loaded conditions in Experiment 1. As in the cued condition, the task names were the same in the study and the test phases. As in the loaded condition, the order of task names was uncorrelated. If foreknowledge of test phase task order invited subjects to coordinate rehearsal of the memory list and performance of the tasks, RTs and switch costs should be greater in the relevant condition than in the irrelevant condition. Previous investigations have shown that foreknowledge of the tasks to be performed reduces RTs overall but does not reduce switch costs; indeed, foreknowledge sometimes increases switch costs (Ruthruff, Remington, \& Johnston, 2001; Sohn \& Anderson, 2001; Sohn \& Carlson, 2000). However, those studies used shorter sequences of tasks, so their results may not generalize to the present experiments.

\section{Method}

Subjects. Twenty-four subjects from the same pool as that in Experiment 1 participated in a single 1-h session for pay or course credit.

Apparatus and Stimuli. The apparatus and stimuli were the same as those in the cued condition in Experiment 1.

Procedure. In both conditions, subjects performed parity and magnitude tasks on digits. The relevant and the irrelevant conditions were tested in separate blocks. In the relevant condition, the procedure was exactly the same as that in the cued condition in Experiment 1 . In the irrelevant condition, the procedure was the same as that in the loaded condition in Experiment 1, except that the task names on the study list were hi-lo and odd-even. There were 40 sets of study-test blocks in each condition, in which each of the lists in Table 1 was presented twice in the study phase and twice in the test phase, in random order. Half of the subjects had the relevant condition before the irrelevant condition, and half had the conditions in the opposite order.

\section{Results}

Test phase. Mean RTs were calculated for correct responses for each subject for repetitions and alternations in Serial Positions 2-6 in each condition. The means across subjects are plotted in Figure 3. The mean percentage of correct responses for each condition is inset in the corresponding bar in Figure 3. Accuracy was high, and there was no evidence of a speed-accuracy trade-off.

Overall, RT was $92 \mathrm{msec}$ longer in the relevant load condition than in the irrelevant load condition, suggesting that the correspondence between the order of task names

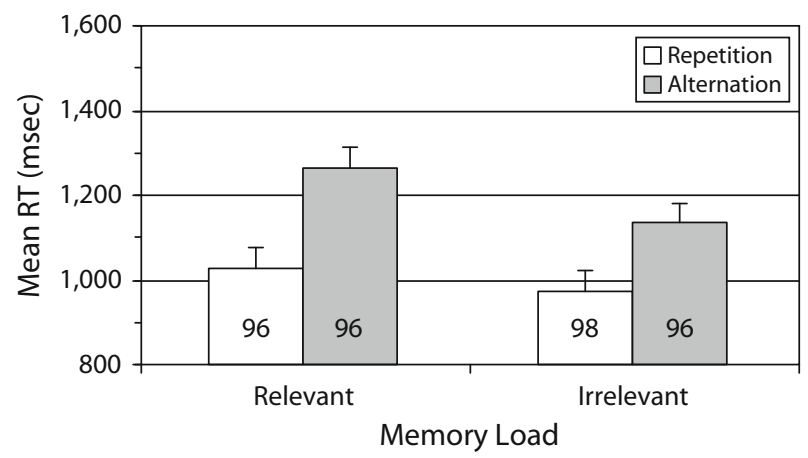

Figure 3. Mean reaction times (RTs) for repetitions and alternations in the relevant load and irrelevant load conditions in Experiment 2 . The error bars represent the $95 \%$ confidence intervals for the means, based on Fisher's least significant difference calculated from the interaction between relevance and repetition. The numbers inside the bars are the percentage of correct responses in the corresponding condition. 
on the study list and the order of tasks (and cues) in the test phase affected performance. Switch costs were also greater in the relevant load condition $(237 \mathrm{msec})$ than in the irrelevant load condition (161 msec), suggesting that the correspondence affected switching, as well as overall RT. A 2 (repetition: repetition or alternation) $\times 2$ (foreknowledge: load relevant or irrelevant) ANOVA on the mean RTs showed a significant main effect of repetition $\left[F(1,23)=61.27, M S_{\mathrm{e}}=15,547.54, p<.01\right]$, indicating switch costs, and a marginally significant main effect of foreknowledge $\left[F(1,23)=3.68, M S_{\mathrm{e}}=55,056.71\right.$, $p=.07]$, indicating slower RTs with a relevant load. The interaction between repetition and foreknowledge was significant $\left[F(1,23)=5.54, M S_{\mathrm{e}}=6,359.45, p<.05\right]$, indicating increased switch costs with foreknowledge of test phase task order (Ruthruff et al., 2001; Sohn \& Anderson, 2001; cf. Sohn \& Carlson, 2000).

Note that mean RT in the relevant condition in this experiment was $120 \mathrm{msec}$ slower than mean RT in the cued condition in Experiment 1, which also required subjects to retain a relevant memory load while performing cued task switching. Two factors may be responsible for this difference. First, the mean RT for the cued condition in Experiment 1 consisted of the averages of mean RTs with the rectangle tasks and the digit tasks, whereas the relevant condition in this experiment included only the digit tasks. In Experiment 1, the rectangle tasks were $106 \mathrm{msec}$ faster than the digit tasks, so the rectangle tasks reduced the overall mean RT. Second, Experiment 1 involved two sessions, whereas this experiment involved only one, so RTs may have been slower because subjects were less practiced. Responses for digit tasks in the cued condition in Experiment 1 were $86 \mathrm{msec}$ faster than those for the digit tasks in the relevant condition in the present experiment, which may be a practice effect.

A 2 (repetition: repetition or alternation) $\times 2$ (foreknowledge: load relevant or irrelevant) ANOVA on the accuracy data showed a main effect of repetition $\left[F(1,23)=21.62, M S_{\mathrm{e}}=3.56, p<.01\right]$. No other effects were significant.

Recall phase. The percentage of study list items recalled correctly in the final recall phase was calculated for each subject, averaging over serial position. The mean recall percentages were $93.8 \%$ and $92.7 \%$ for the relevant and the irrelevant conditions, respectively. A one-way ANOVA comparing recall accuracy in the relevant and the irrelevant conditions showed that the difference was not significant.

\section{Discussion}

The RT data suggest that a correlation between the order of task names in the study list and cues in the test list may invite subjects to coordinate the study list with the test list. Several ways of coordinating study lists and test lists will be considered in the General Discussion section. For now, the data indicate that plan-level processing affected tasklevel processing, increasing RTs and switch costs in the relevant condition, as compared with the irrelevant condition (cf. Ruthruff et al., 2001; Sohn \& Anderson, 2001; Sohn \& Carlson, 2000).

\section{EXPERIMENT 3 Content-Specific Interference and Switch Costs}

The cued and the loaded conditions in Experiment 1 differed in their potential to produce content-specific interference, as well as in foreknowledge of the sequence of tasks to be performed. In the cued condition, the items in the memory list were the same as the cues that were used in the test phase. For example, in one session with the cued condition, the memory list items were hi-lo and odd-even, and the test phase cues were also hi-lo and odd-even. The memory list items could interfere with encoding of the test phase cues, and the test phase cues could interfere with retention of the memory items. Plan-level processing may have been engaged to reduce this interference, and that may have inflated RTs and switch costs. By contrast, in the loaded condition, the memory items were different from the test phase cues. For example, in one session with the loaded condition, the memory list items were wide-narrow and red-green and the test phase cues were $h i-l o$ and odd-even. These memory list items would be less likely to interfere with cue encoding, and these cues would be less likely to interfere with retention of the memory list. Plan-level processing would not have to be engaged to reduce interference, so RTs and switch costs may have been smaller in the loaded condition than in the cued condition.

In Experiment 3, the effects of content-specific interference in the absence of foreknowledge were examined by manipulating the similarity between the memory list items and the test phase cues. The experiment tested the hypothesis that RTs and switch costs would be greater when the items on the memory list were the same as the cues in the test phase than when the items on the memory list were different from the cues in the test phase. Subjects performed cued task switching with a concurrent memory load that did not provide foreknowledge of the order of the tasks in the test phase. In two conditions, the identities of the items in the memory list were the same as the identities of the cues in the test phase, creating the potential for content-specific interference that would increase RTs and switch costs. In the other two conditions, the identities of the items in the memory list were different from the identities of the cues in the test phase, reducing the potential for content-specific interference, so there would be no increase in RTs and switch costs.

To implement this design, two types of cues were used to refer to each task: name cues and mapping cues. Contentspecific interference was expected when the memory items and the test phase cues were of the same type (both name cues or both mapping cues). Less content-specific interference was expected when the memory items and the test phase cues were of different types (name cues in the memory list and mapping cues in the test phase or vice versa). The name cues were single words (parity and magnitude) that named the task to be performed without specifying the response categories or the mapping of response categories onto response keys. The mapping cues were pairs of words (odd-even and $h i-l o$ ) that specified the response categories and the mapping of response cat- 
egories onto response keys (Mayr \& Kliegl, 2000). A factorial combination of cue type and study versus test phase produced four different conditions: mapping-mapping, in which mapping cues were used in the study phase and the test phase; name-name, in which name cues were used in the study phase and the test phase; name-mapping, in which name cues were used in the study phase and mapping cues were used in the test phase; and mapping-name, in which mapping cues were used in the study phase and name cues were used in the test phase. There should be more content-specific interference in the mappingmapping and name-name conditions than in the namemapping and mapping-name conditions. In all four conditions, the order of the cues in the study list was independent of the order of the cues in the test list, so the memory list provided no foreknowledge of task order in the test phase.

Experiment 3 also provided a comparison of test phase RT with mapping cues and name cues. Mayr and Kliegl (2000) found that RT was substantially faster with mapping cues than with name cues, and they used this difference to support their claim that subjects retrieve mapping rules in response to cues in the explicit task-cuing procedure. The mapping rules are provided directly by the mapping cues, but they have to be retrieved with name cues. The difference in RT between mapping and name cues provides an estimate of the time required to retrieve mapping rules. However, Mayr and Kliegl (2000) compared meaningful words as mapping cues with arbitrary symbols as name cues, and this confounds the transparency of the cue with the requirement to retrieve mapping rules. Transparent cues, such as words that name the task or the response categories, produce smaller switch costs than do arbitrary cues, such as unrelated letters or colors or shapes (Logan \& Bundesen, 2004). The difference Mayr and Kliegl (2000) observed may have been due to the transparency of the cues, rather than to the requirement to retrieve mapping rules. The present experiment allowed a comparison of name and mapping cues with transparent cues (i.e., words whose conventional meanings named the tasks or the response categories).

\section{Method}

Subjects. Twenty-four subjects from the same pool participated in two 1-h sessions for pay.

Apparatus and Stimuli. The cues were the words parity, magnitude, odd-even, and hi-lo, and the targets were the digits 1-9, excluding 5. Otherwise, the apparatus and stimuli were the same as those in the cued condition in Experiment 1.

Procedure. The procedure was the same as that in the irrelevant load condition in Experiment 2. Subjects saw a list of cues to remember, followed by a list of targets and cues to respond to. The order of cues in the memory list and the order of tasks on the test list were sampled independently from the set of 20 possible orders in Table 1. Thus, the memory list was irrelevant to the test phase, in that it provided no foreknowledge of the order of tasks in the test phase. There were 40 sets of study, test, and recall trials in each condition.

There were four main conditions, defined by the relation between the type of cues used in the memory lists and the type of cues used in the test phase: mapping-mapping, name-name, name-mapping, and mapping-name. The four conditions were run in two 1-h sessions, with two conditions in each session. The order of the conditions was counterbalanced between subjects with a $4 \times 4$ Latin square. Six subjects received each order of conditions.

\section{Results}

Test phase. Mean RTs were calculated for Serial Positions 2-6 in the test phase for each subject, separating repetitions from alternations. The means across subjects in each of the four conditions appear in Figure 4. Accuracy was high, and there was no evidence of a speed-accuracy trade-off. There were large switch costs in each condition, averaging $223 \mathrm{msec}$. RT was faster with name cues than with mapping cues, following Mayr and Kliegl (2000), but the difference was only $24 \mathrm{msec}$. RT was longer (by $76 \mathrm{msec}$ ) and switch costs were greater (by $36 \mathrm{msec}$ ) when study and test cue types matched (i.e., mappingmapping and name-name) than when they mismatched (i.e., mapping-name and name-mapping), indicating a content-specific interference effect.

These conclusions were evaluated in a 2 (repetition: repetition or alternation) $\times 2$ (study cues: name or mapping) $\times 2$ (test cues: name or mapping) ANOVA on the mean RTs. The main effect of repetition was significant $\left[F(1,23)=74.24, M S_{\mathrm{e}}=32,123.73, p<.01\right]$, but the main effects for study cues $[F(1,23)<1]$, and test cues $[F(1,23)<1]$ were not significant. The interaction between study cues and test cues was significant $[F(1,23)=$ $\left.12.35, M S_{\mathrm{e}}=22,696.43, p<.01\right]$, as was the interaction between study cues, test cues, and repetition $[F(1,23)=$ $\left.5.14, M S_{\mathrm{e}}=2,989.10, p<.05\right]$. The nonsignificant main effect of test cues fails to replicate Mayr and Kliegl's (2000) results, suggesting that their effect was due to the transparency of the cues, rather than to retrieval of mapping rules. The significant interaction between study cues and test cues suggests that content-specific interference may affect overall RT, and the significant interaction between study cues, test cues, and repetition suggests that content-specific interference affects switch costs.

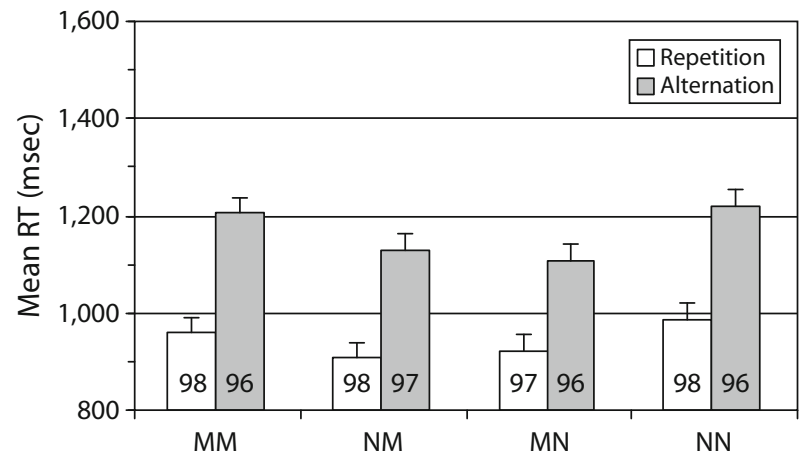

Figure 4. Mean reaction times (RTs) for repetitions and alternations for each combination of name cues $(N)$ and mapping cues (M) in the study list and test list. The error bars represent the $\mathbf{9 5 \%}$ confidence intervals for the means, based on Fisher's least significant difference calculated from the interaction between repetition, cues at study, and cues at test. The numbers inside the bars are the percentage of correct responses in the corresponding condition. 
A 2 (repetition: repetition or alternation) $\times 2$ (study cues: name or mapping) $\times 2$ (test cues: name or mapping) ANOVA on the accuracy data yielded a significant main effect of repetition $\left[F(1,23)=19.12, M S_{\mathrm{e}}=6.98\right.$, $p<.01]$. No other effects were significant.

Recall phase. The percentage of study list items recalled correctly was averaged over serial position for each subject. The means across subjects were $94.9 \%, 95.1 \%$, $95.3 \%$, and $95.9 \%$ for the mapping-mapping, namename, mapping-name, and name-mapping conditions, respectively. A 2 (study cues: name or mapping) $\times 2$ (test cues: name or mapping) ANOVA on the recall probabilities yielded no significant effects.

\section{Discussion}

Switch costs were larger and RTs were slower when the same kind of cues appeared in the study phase and the test phase, as compared with switch costs and RTs when the cues were different. This suggests that switch costs were affected by content-specific interference (cf. Allport, Styles, \& Hsieh, 1994; Allport \& Wylie, 2000; Waszak, Hommel, \& Allport, 2003; Wylie \& Allport, 2000). The interference could have affected the time required to encode the cues in the study phase, the time devoted to rehearsing the memory list during the test phase, or both processes. These alternatives will be discussed in greater detail in the General Discussion section.

\section{EXPERIMENT 4 Explicit Cuing With and Without a Memory Load}

None of the previous experiments included a control condition in which there was no memory load in order to evaluate the effect of loading memory on switch costs. The final experiment compared performance with and without a concurrent memory load in the explicit taskcuing procedure. In the loaded (dual-task) condition, subjects received a memory load of six irrelevant task names, as in the loaded condition in Experiment 1. Then they performed six cued trials, after which they recalled the memory load. In the unloaded (single-task) condition, subjects received a memory load and recalled it immediately before performing the six cued trials. Thus, the unloaded condition was like the loaded condition, except for the order of the phases. The unloaded condition involved study, recall, and test phases, whereas the loaded condition involved study, test, and recall phases.

\section{Method}

Subjects. Two groups of 24 subjects served in a single 1-h session for course credit or pay.

Apparatus and Stimuli. The apparatus and stimuli were the same as those in the previous experiments.

Procedure. Half of the subjects had red-green and widenarrow as memory load items, hi-lo and odd-even as cues, and digits as targets. The other half had hi-lo and odd-even as memory load items, red-green and wide-narrow as cues, and colored rectangles as targets. In the loaded condition, the procedure was the same as that in the loaded condition in Experiment 1. Subjects received a memory load of six task names. Next they performed six cued trials.
Then they recalled the memory load in response to recall prompts. In the unloaded condition, subjects received a memory load of six task names. Next they recalled it in response to recall prompts. Then they performed six cued trials. Each subject received 40 lists in the loaded condition and 40 lists in the unloaded condition. Half of the subjects received the loaded condition before the unloaded condition, and half received the conditions in the opposite order.

\section{Results}

Test phase. The mean RTs for repetitions and alternations are plotted separately for loaded and unloaded conditions in Figure 5. The percentage of correct responses in each condition is inset in the corresponding bar in the figure. Accuracy was high, and there was no evidence of a speed-accuracy trade-off. As was expected, RT was faster overall for repetitions $(M=1,013 \mathrm{msec})$ than for alternations ( $M=1,234 \mathrm{msec})$ and faster overall in the unloaded (single-task) condition $(M=1,067 \mathrm{msec})$ than in the loaded (dual-task) condition $(M=1,180 \mathrm{msec})$. However, memory load had little effect on the difference between repetitions and alternations. Switch costs were essentially the same in the loaded and the unloaded conditions ( $M \mathrm{~s}=227$ and $214 \mathrm{msec}$, respectively). These conclusions were supported by a 2 (repetition: repetition or alternation) $\times 2$ (load: loaded or unloaded) $\times 2$ (target: digits or rectangles) ANOVA on the mean RTs. The only significant effects in the ANOVA were the main effects of repetition $\left[F(1,46)=112.46, M S_{\mathrm{e}}=20,723.79, p<.01\right]$ and load $\left[F(1,46)=6.02, M S_{\mathrm{e}}=102,416.40, p<.05\right]$. The interaction between repetition and load was not significant $\left[F(1,46)=0.44, M S_{\mathrm{e}}=5,335.53, p=.511\right]$, nor was the interaction between repetition, load, and target $\left[F(1,46)=2.13, M S_{\mathrm{e}}=5,335.53, p=.151\right]$.

The null interaction between repetition and load is important theoretically, so a power analysis was conducted to determine the number of subjects that would be required to achieve significance at $p<.05$ with a probability (power) of .80. The effect size for the interaction, expressed as Cohen's (1988) $d$, was .094, which is a very small value. If significance were assessed with a paired-sample $t$ test (which is equivalent to the $F$ test that was used to evalu-

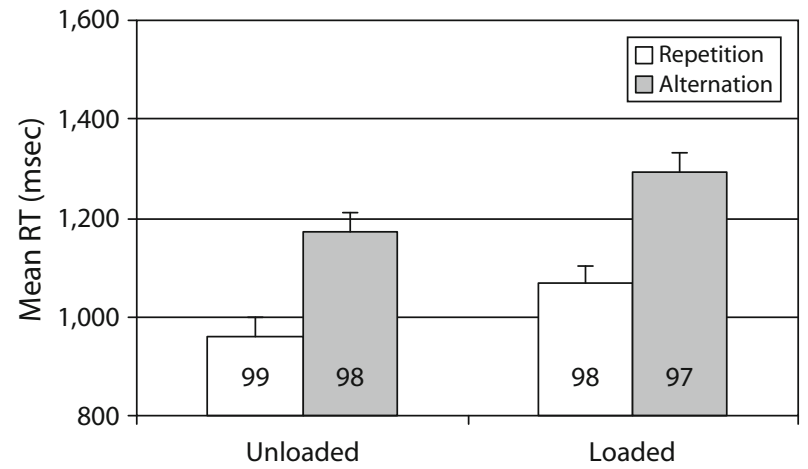

Figure 5. Mean reaction times (RTs) for repetitions and alternations in loaded (dual-task) and unloaded (single-task) conditions. The error bars represent the $\mathbf{9 5 \%}$ confidence intervals for the means, based on Fisher's least significant difference calculated from the interaction between repetition and load. 
ate the interaction in the ANOVA), 720 subjects would be required to achieve significance at $p<.05$ with a probability of .80 if the test was one-tailed and 900 subjects to achieve the same significance level with the same probability if the test was two-tailed. These are inordinately large numbers of subjects, especially for a within-subjects design, so it seems reasonable to conclude that the interaction is null.

A 2 (repetition: repetition or alternation) $\times 2$ (load: loaded or unloaded) $\times 2$ (target: digits or rectangles) ANOVA on the accuracy data yielded a significant main effect of repetition $\left[F(1,46)=10.16, M S_{\mathrm{e}}=3.28\right.$, $p<.01]$. No other effects were significant.

Recall phase. The probability of recalling study list items correctly in the recall phase was averaged over each position for each subject. The means across subjects were $91.4 \%$ in the loaded condition and $94.4 \%$ in the unloaded condition. A 2 (load: loaded or unloaded) $\times 2$ (target: digits or rectangle) ANOVA on the recall probabilities revealed no significant effects.

\section{Discussion}

Memory load and repetition had substantial effects on RT, but there was no interaction between them. Switch costs were the same in the loaded and the unloaded conditions. This suggests that memory load did not contribute to switch costs for in the previous experiments on explicit task cuing. However, it is possible that memory load may be partly responsible for the larger switch costs for the task span procedure in Experiment 1 (but see Logan, 2004, 2006).

\section{GENERAL DISCUSSION}

Four experiments were conducted to examine the interaction between plan-level and task-level processing and its contribution to switch costs. Experiment 1 compared the task span procedure, which involves several plan-level processes, with two versions of the explicit task-cuing procedure, which involve fewer plan-level processes. Switch costs were greater for the task span procedure than for either version of the explicit task-cuing procedure, suggesting that coordinating retrieval of task names with target processing, protecting against content-specific interference between the plan and the tasks, maintaining the plan in memory, or some combination of these processes may inflate switch costs. Experiments 2-4 were performed to evaluate these possibilities.

Experiment 2 compared versions of the explicit taskcuing procedure in which subjects held in memory lists of task names whose order did or did not correspond to the order of cues in the test trials and showed larger switch costs when order corresponded. This suggests that coordination of the memory list with target processing increases switch costs. Experiment 3 compared versions of the explicit task-cuing procedure in which subjects held in memory lists of task names that were similar or dissimilar to the cues presented in the test trials. Switch costs were greater when the memorized names and cues were similar than when they were dissimilar, suggesting that content- specific interference increases switch costs. Experiment 4 compared explicitly cued performance with and without a concurrent memory load and showed no effect of memory load on switch costs, suggesting that loading memory per se does not contribute to switch costs, although it increases RT substantially.

\section{Cue-Encoding Benefits or True Switch Costs?}

Experiments 2-4 demonstrate that coordinating lists and targets and protecting against content-specific interference contribute to switch costs in the present versions of the explicit task-cuing procedure, but another step in logic is required to infer that these processes also contribute to switch costs in the task span procedure. That inference requires the assumption that the same mechanism produces switch costs in the task span procedure and the explicit task-cuing procedure. Recent studies of the explicit task-cuing procedure have suggested that the mechanisms may not be identical (Logan \& Bundesen, 2003; Mayr \& Kliegl, 2003). They have noted that most explicit taskcuing procedures, including the present ones, use only one cue for each task, which confounds cue repetition with task repetition. The cue repeats whenever the task repeats, and the cue switches whenever the task switches. Thus, task repetitions may be faster than task alternations because cue encoding benefits from repetition. In the recent studies, cue-encoding benefits have been separated from "true switch costs" through the use of two cues for each task, so there are three kinds of transitions between tasks, instead of the usual two: cue repetitions, in which the cue and the task both repeat (e.g., hi-lo $\rightarrow$ hi-lo); task repetitions, in which the cue switches but the task repeats (e.g., magnitude $\rightarrow$ hi-lo); and task alternations, in which the cue and the task both switch (e.g., odd-even $\rightarrow$ $h i-l o$ ). Cue-encoding benefits are assessed by comparing cue repetitions with task repetitions. True switch costs are assessed by comparing task repetitions with task alternations. Several experiments have shown substantial cue-encoding benefits, and many of them have shown significant switch costs (Arrington \& Logan, 2004; Logan \& Bundesen, 2003, 2004; Logan \& Schneider, 2006; Mayr \& Kliegl, 2003; Schneider \& Logan, 2005, 2006).

The present Experiments 2-4 included only cue repetitions and task alternations, so some component of the reported switch costs (the difference between cue repetitions and task alternations) may reflect cue-encoding benefits. In order to generalize the conclusions from Experiments 2-4 to Experiment 1, it must be assumed that the correlation between lists and tasks and the similarity of lists and cues affected true switch costs, and not cueencoding benefits. The data are consistent with this assumption. If these manipulations increased switch costs by increasing cue-encoding benefits, they should have reduced RT for task repetitions without affecting RT for task alternations. Instead, they increased RT for task repetitions and increased it even more for task alternations (see Figures 3 and 4), which suggests that they affected the true switch cost component.

An alternative interpretation, suggested by an anonymous reviewer, is that cue-encoding benefits in the ex- 
plicit task-cuing procedure are really cue-encoding costs. Task repetitions may be slower than cue repetitions because changing the cue produces a cost, not because repeating the cue produces a benefit. This interpretation suggests that the increased switch costs in Experiments 2-4 may reflect increased cue-encoding costs, rather than increased true switch costs. So far, no one has run the neutral conditions that would be necessary to separate cue-encoding benefits from cue-encoding costs-indeed, it is hard to conceive cues that would be neutral and still cue the appropriate tasks-but there are empirical and theoretical reasons to believe that the effects are cue-encoding benefits, rather than costs. Empirically, priming manipulations generally produce strong benefits and rarely produce robust costs (for a review, see McNamara, 2005). Theoretically, Logan and Bundesen (2003) and Schneider and Logan (2005) successfully modeled time course functions for the various transitions by assuming that cue encoding depends on the matching of representations of past cues in short-term memory and semantic representations in long-term memory. They assumed that the matches to short-term and long-term memory race against each other and that the cue is encoded when one of the traces matches. Thus, encoding would be faster when the current cue matches both short-term and long-term memory representations than when it matches only long-term memory representations. Short-term memory representations would match the current cue only on cue repetition trials, so only cue repetitions would benefit from the race between short-term and long-term memory matches. Short-term memory representations would not match the current cue on task-repetition and task-alternation trials, so cue encoding would not benefit from repetition.

Generalization from Experiments 2-4 to the task span condition in Experiment 1 also requires the assumption that true switch costs are produced by the same mechanism in the explicit task-cuing procedure and the task span procedure. Although the experiments provide no direct evidence for this assumption, the assumption that different task-switching procedures capture the same taskswitching mechanism is common in the task-switching literature (Logan, 2003; Monsell, 2003). The different procedures are viewed as providing converging evidence for the same executive control processes (Gilbert \& Shallice, 2002; Logan \& Gordon, 2001; Meiran, 2000).

\section{Toward a Model of Plan-Level and Task-Level Processing}

To account for performance in the task span procedure, a model of serial memory must be combined with a model of task switching. There are several models of each kind available in the literature, so there are many possible combinations. I prefer to combine Anderson and Matessa's (1997) ACT-R model of serial memory with Schneider and Logan's (2005) priming model of task switching. Both models have rich legacies dating back many years, and both have formal architectures that enable computer simulation and mathematical modeling. The combination is particularly attractive because the formal architectures are closely related (see Anderson \& Betz, 2001; Logan, 2002; Nosofsky, 1991) and the processes are complementary: One provides input to the other.

Anderson and Matessa's (1997) model accounts for serial recall in terms of a production system that retrieves lists from memory, chunks from lists, and items from chunks. The production system is driven by activation, which predicts the speed and accuracy of retrieval as a function of practice, recency, and the number of competing alternatives. Schneider and Logan's (2005) model accounts for task switching in terms of a retrieval process in which a representation of the task cue is combined with a representation of the target to form a compound retrieval cue that pulls an appropriate response from memory. Speed and accuracy depend on the ease with which task cue and target representations can be formed and combined into a compound retrieval cue and on the state of the subsequent retrieval process, and processing is generally faster when tasks repeat. Applied to the task span procedure, Anderson and Matessa's model describes plan-level processing, and Schneider and Logan's (2005) model describes tasklevel processing. Anderson and Matessa's model encodes memory lists in the study phase, maintains the list, and retrieves successive task names in the test phase and the final recall phase. The task names it retrieves in the test phase are input as cues to Schneider and Logan's (2005) model, which retrieves the appropriate response to each target. Developing a formal model that instantiates these ideas is beyond the scope of this article. For one thing, the number of parameters would approach the number of data points. Nevertheless, the sketch of the model drawn here may provide some insights into the results of the experiments. The insights are provisional and may be predicted by other combinations of memory models and taskswitching models that have not yet been proposed.

Keeping track of task order. Experiments 1 and 2 suggest that the larger switch costs and longer RTs for the task span procedure and in the cued condition were due, in part, to the correspondence between the order of task names on the memory list and the order of tasks to be performed in the test phase. The reason for the increased RTs for the task span procedure is straightforward: Subjects had to retrieve the next task in the memory list in order to know how to process the corresponding target. Retrieval takes time (Anderson \& Matessa, 1997), and this would increase test phase RT. It is less clear why retrieval from the memory list would increase switch costs, since retrieval is required whether the task repeats or alternates. The provisional model suggests several possibilities. First, retrieval may be faster when the item to be retrieved repeats than when it alternates because there is facilitation from just having retrieved the same item or because there is less interference from the previously retrieved item. Indeed, Logan (2006) found faster retrieval for repeated items in a memory span task. This speeded retrieval would increase switch costs by reducing test phase RTs on repetition trials. Second, the retrieved task name must be combined with the target to form a compound retrieval cue that pulls the required response from memory. The formation of the compound retrieval cue may be facilitated by repeating 
the task, and this would increase switch costs by reducing RTs on repetition trials. Third, subjects may rehearse the memory list items in the test phase as they recall them, in order to keep them available in memory for the final recall phase. Rehearsal may contribute to test phase RT, and rehearsal may be faster for repeated items, which would increase switch costs by speeding RT on repetition trials. Further research will be required to assess the validity of each of these possibilities.

It is less clear why correspondence between the order of task names in the memory list and cues in the test phase should increase RTs and switch costs in the cued condition in Experiment 1 and the relevant condition in Experiment 2 . The memory lists provided foreknowledge of the tasks to be performed, and previous research has shown that foreknowledge reduces RT overall, although it sometimes increases switch costs (Ruthruff et al., 2001; Sohn \& Anderson, 2001; Sohn \& Carlson, 2000). Again, the provisional model suggests several possibilities. First, the correspondence between the order of the memory lists and the order of the tasks may invite subjects to step through the memory list in the test phase in order to gain foreknowledge of the task to be performed. This may seem unlikely, because the cues presented on each test phase trial specify the task to be performed and because RT clearly did not benefit from foreknowledge. However, subjects' confidence in the tasks they are performing may increase by establishing correspondence, and that may be enough to motivate them to do it. Second, subjects may retrieve the task names in the memory list when they encounter the corresponding cues, in order to rehearse the memory list in preparation for the final recall phase. There was little difference in final recall accuracies between the loaded condition and the other conditions in Experiment 1 and between the relevant and the irrelevant conditions in Experiment 2 , so this strategy may not have increased accuracy. Nevertheless, it may have increased subjects' confidence, and that may have motivated them to do it. Third, as has been suggested by reviewer Eric Ruthruff, subjects may use the test phase as an opportunity to learn the memory list, and this would add to the time required for task performance. This learning could supplement their learning in the study phase, or it could replace it entirely: Subjects could ignore the memory list in the study phase and then learn it in the test phase. Each of these strategies would increase RT overall, and each of them could increase switch costs, because retrieval, rehearsal, and learning may be faster for repeated items than for alternated items. Further research will be required to evaluate these possibilities.

Protecting against content-specific interference. Experiment 3 suggests that the larger switch costs in the task span and cued conditions in Experiment 1 may have been due, in part, to the overlap between the items in the memory list and the tasks and cues in the test phase. Experiment 3 showed that RT and switch costs were larger when the items in the memory list and the cues in the test phase were the same than when they were different, even when the orders of the memory list items and the test phase tasks were unrelated. The provisional model would account for these effects in terms of interference. It is well established that interference increases with similarity between items (Anderson \& Matessa, 1997), so there would be more interference between the memory items and the task cues when they were similar (in the mappingmapping and name-name conditions) than when they were dissimilar (in the mapping-name and namemapping conditions). The greater interference could have several effects on performance. It would slow retrieval of items from the memory list, prolong rehearsal and maintenance of the memory items, slow encoding of cues presented in the test phase, and slow retrieval of appropriate responses to the test phase targets. All of these factors would increase RT. Interference would be greater when tasks switched than when they repeated. In Anderson and Matessa's model, the most recent items have the greatest impact on performance, so interference would be greater and RT would be longer when the last item was different from the current one (i.e., on task alternation trials). Again, further research will be required to evaluate these possibilities.

Effects of concurrent memory load. Experiment 4 suggested that merely retaining a load in working memory does not interact with the task-level processes that produce switch costs. This conclusion is consistent with many previous investigations of the effects of concurrent memory load. Egeth (1977) and Logan (1978) found that memory load did not interact significantly with factors that affected several stages of RT tasks, including stimulus degradation, stimulus-response compatibility, and the number of items to be examined in visual search, although memory load increased overall RT in each experiment. Recent experiments by Woodman, Vogel, and Luck (2001), Woodman and Luck (2004), and Oh and Kim (2004) replicated these null interactions with the number of items in a visual search display and showed that interactions could be produced only by concurrent tasks that affected executive processing, as well as loading working memory.

The provisional model would account for the main effect of memory load in terms of plan-level processes that maintain the memory list. There would be no interaction with switch costs, because the memory lists differ from the tasks in the test phase in identity and order, so there is no need to protect against content-specific interference or to establish correspondence between items in the memory list and tasks in the test phase.

\section{Conclusions}

The novel contribution of the present experiments is to distinguish between plan-level and task-level executive control processes empirically and theoretically. They provide evidence of the hierarchical control structures envisioned by Miller et al. (1960), in which plans control subordinate processing, and they show that implementing a plan produces predictable costs that affect task-level processing. They provide ways to extend research on switching between simple tasks to broader contexts, generalizing concepts of control from simple prepared reflexes (Hommel, 2000; Logan, 1978) to complex sequences of 
thought and action, such as those involved in finishing your business at the office and going home to make dinner.

\section{AUTHOR NOTE}

This research was supported by National Science Foundation Grants BCS 0133202 and BCS 0446806. I am grateful to Julie Delheimer for testing the subjects and analyzing the data, to Darryl Schneider for helpful comments on the manuscript and valuable discussions about the costs and benefits of implementing plans, and to Andy Tomarken for helping with the power analysis in Experiment 4. Correspondence concerning this article may be addressed to G. D. Logan, Department of Psychology, Vanderbilt University, Nashville, TN 37203 (e-mail: gordon.logan@vanderbilt.edu).

\section{REFERENCES}

Allport, D. A., Styles, E. A., \& Hsieh, S. (1994). Shifting intentional set: Exploring the dynamic control of tasks. In C. Umiltà \& M. Moscovitch (Eds.), Attention and performance XV: Conscious and nonconscious information processing (pp. 421-452). Cambridge, MA: MIT Press, Bradford Books.

Allport, D. A., \& Wylie, G. R. (2000). Task switching, stimulusresponse bindings, and negative priming. In S. Monsell \& J. Driver (Eds.), Attention and performance XVIII: Control of cognitive processes (pp. 35-70). Cambridge, MA: MIT Press.

ANDERSON, J. R., \& BETZ, J. (2001). A hybrid model of categorization. Psychonomic Bulletin \& Review, 8, 629-647.

Anderson, J. R., \& Matessa, M. P. (1997). A production system theory of serial memory. Psychological Review, 104, 728-748.

Arrington, C. M., \& Logan, G. D. (2004). Episodic and semantic components of the compound-stimulus strategy in the explicit task-cuing procedure. Memory \& Cognition, 32, 965-976.

COHEN, J. (1988). Statistical power analysis for the behavioral sciences (2nd ed.). Hillsdale, NJ: Erlbaum.

EGETH, H. (1977). Attention and preattention. In G. H. Bower (Ed.), The psychology of learning and motivation (Vol. 11, pp. 277-320). New York: Academic Press.

Gilbert, S. J., \& Shallice, T. (2002). Task switching: A PDP model. Cognitive Psychology, 44, 297-337.

Hayes-Roth, B., \& Hayes-Roth, F. (1988). A cognitive model of planning. In A. M. Collins \& E. E. Smith (Eds.), Readings in cognitive science: A perspective from psychology and artificial intelligence (pp. 496-513). San Mateo, CA: Morgan Kaufmann.

Hommel, B. (2000). The prepared reflex: Automaticity and control in stimulus-response translation. In S. Monsell \& J. Driver (Eds.), Attention and performance XVIII: Control of cognitive processes (pp. 247273). Cambridge, MA: MIT Press.

Kotovsky, K., Hayes, J. R., \& Simon, H. A. (1985). Why are some problems hard? Evidence from Tower of Hanoi. Cognitive Psychology, 17, 248-294.

LogAN, G. D. (1978). Attention in character classification: Evidence for the automaticity of component stages. Journal of Experimental Psychology: General, 107, 32-63.

Logan, G. D. (2002). An instance theory of attention and memory. Psychological Review, 109, 376-400.

LogAN, G. D. (2003). Executive control of thought and action: In search of the wild homunculus. Current Directions in Psychological Science, 12, 45-48.

Logan, G. D. (2004). Working memory, task switching, and executive control in the task span procedure. Journal of Experimental Psychology: General, 133, 218-236.

Logan, G. D. (2006). Out with the old, in with the new: More valid measures of switch cost and retrieval time in the task span procedure. Psychonomic Bulletin \& Review, 13, 139-144.

LogAN, G. D., \& BundesEn, C. (2003). Clever homunculus: Is there an endogenous act of control in the explicit task-cuing procedure? Journal of Experimental Psychology: Human Perception \& Performance, 29, 575-599.
Logan, G. D., \& Bundesen, C. (2004). Very clever homunculus: Compound stimulus strategies for the explicit task-cuing procedure. Psychonomic Bulletin \& Review, 11, 832-840.

LOGAN, G. D., \& GORDON, R. D. (2001). Executive control of visual attention in dual-task situations. Psychological Review, 108, 393-434.

Logan, G. D., \& SchNeIDER, D. W. (2006). Priming or executive control? Associative priming of cue encoding increases "switch costs" in the explicit task-cuing procedure. Memory \& Cognition, 34, 1250-1259.

Mayr, U., \& Kliegl, R. (2000). Task-set switching and long-term memory retrieval. Journal of Experimental Psychology: Learning, Memory, \& Cognition, 26, 1124-1140.

MaYr, U., \& KLIEGL, R. (2003). Differential effects of cue changes and task changes on task-set selection costs. Journal of Experimental Psychology: Learning, Memory, \& Cognition, 29, 362-372.

McNamara, T. P. (2005). Semantic priming: Perspectives from memory and word recognition. New York: Psychology Press.

Meiran, N. (1996). Reconfiguration of processing mode prior to task performance. Journal of Experimental Psychology: Learning, Memory, \& Cognition, 22, 1423-1442.

Meiran, N. (2000). Reconfiguration of stimulus task sets and response task sets during task switching. In S. Monsell \& J. Driver (Eds.), Attention and performance XVIII: Control of cognitive processes (pp. 377-399). Cambridge, MA: MIT Press.

Miller, G. A., Galanter, E., \& Pribram, K. H. (1960). Plans and the structure of behavior. New York: Holt.

Monsell, S. (2003). Task switching. Trends in Cognitive Sciences, 7 , 134-140.

NosofsKy, R. M. (1991). Relation between the rational model and the context model of classification. Psychological Science, 2, 416-421.

Он, S.-H., \& Kıм, M.-S. (2004). The role of spatial working memory in visual search efficiency. Psychonomic Bulletin \& Review, 11, 275-281.

Ruthruff, E., Remington, R. W., \& Johnston, J. C. (2001). Switching between simple cognitive tasks: The interaction of top-down and bottom-up factors. Journal of Experimental Psychology: Human Perception \& Performance, 27, 1404-1419.

Schneider, D. W., \& Logan, G. D. (2005). Modeling task switching without switching tasks: A short-term priming account of explicitly cued performance. Journal of Experimental Psychology: General, 134, 343-367.

Schneider, D. W., \& Logan, G. D. (2006). Priming cue encoding by manipulating transition frequency in explicitly cued task switching. Psychonomic Bulletin \& Review, 13, 145-151.

SoHN, M.-H., \& ANDERSON, J. R. (2001). Task preparation and task repetition: Two-component model of task switching. Journal of Experimental Psychology: General, 130, 764-778.

SoHN, M.-H., \& CARLSON, R. A. (2000). Effects of repetition and foreknowledge in task-set reconfiguration. Journal of Experimental Psychology: Learning, Memory, \& Cognition, 26, 1445-1460.

Sudevan, P., \& TAYlor, D. A. (1987). The cuing and priming of cognitive operations. Journal of Experimental Psychology: Human Perception \& Performance, 13, 89-103.

Thomas, J. C. (1974). An analysis of behavior in the hobbits-orcs problem. Cognitive Psychology, 6, 257-269.

WaszaK, F., Hommel, B., \& Allport, A. (2003). Task-switching and long-term priming: Role of episodic stimulus-task bindings in taskshift costs. Cognitive Psychology, 46, 361-413.

Woodman, G. F., \& LUCK, S. J. (2004). Visual search is slowed when visuospatial working memory is occupied. Psychonomic Bulletin \& Review, 11, 269-274.

Woodman, G. F., Vogel, E. K., \& Luck, S. J. (2001). Visual search remains efficient when visual working memory is full. Psychological Science, 12, 219-224.

Wylie, G., \& Allport, A. (2000). Task switching and the measurement of "switch costs." Psychological Research, 63, 212-233.

(Manuscript received October 12, 2005; revision accepted for publication February 15, 2006.) 